\title{
Quand l'environnement relationnel contribue à la compréhension du burnout
}

\author{
par Chantal Fuhrer ${ }^{133}$, Virginie Moisson ${ }^{134}$ \\ et Alain Cucchi ${ }^{135}$
}

Résumé

Cette recherche a pour objectif d'étudier les liens entre l'environnement relationnel perçu d'un individu et une forme de rupture psychologique qu'est l'épuisement professionnel ou burnout. L'environnement relationnel est abordé sous l'angle des relations avec l'organisation, des relations avec l'entourage ainsi que des relations avec le couple travail-famille. L'épuisement professionnel, au sens de Maslach, comprend l'assèchement émotionnel, le cynisme et l'efficacité professionnelle. Pour traiter cette problématique, un échantillon de 639 infirmières est analysé. L'étude exploratoire présente un modèle structurel qui est testé. Les résultats montrent l'existence de liens significatifs entre l'environnement relationnel et l'épuisement professionnel, avec des facteurs explicatifs différents. Les principaux résultats sont analysés avant de conclure sur des pistes de recherches futures.

\section{Abstract}

This research aims to study the relationship between perceived relational environment and the burnout which can be considered as a kind of psychological break. Relational environment includes relations towards the organization, relations towards circle of acquaintances and relations towards the couple job/family. The three dimensions of Maslach burnout are used: exhaustion, cynicism and inefficacy. A sample of 639 nurses is analysed. The exploratory study presents a structural model which is tested. Results show significant ties between relational environment and the burnout, with different degree of signification. Main results are analysed before we conclude with future research perspective.

Le stress ou syndrome général d'adaptation, dans l'approche biologique de Selye (1975) est bénéfique à l'organisme jusqu'à ce qu'il se transforme en pathologie. Si celui-ci est ponctuel et limité dans le temps, il s'avère moteur. Par contre, s'il perdure, l'individu entre dans un état d'anxiété chronique pouvant entraîner des troubles potentiellement graves. Dans ce cas, une véritable rupture psychologique

\footnotetext{
133. Chantal Fuhrer, IAE, Université de la Réunion, CEMOI (Centre d'Economie et de Management de l'Océan Indien), chantal.fuhrer@univ-reunion.fr

134. VIRGINIE Moisson, IAE, Université de la Réunion, CEMOI (Centre d'Economie et de Management de l'Océan Indien), virginie.moisson@univ-reunion.fr

135. Alain Cucchi, IAE, Université de la Réunion, CEMOI (Centre d'Economie et de Management de l'Océan Indien), alain.cucchi@univ-reunion.fr
} 
s'opère et ce décrochage peut entraîner le burnout (syndrome d'épuisement), phase aggravée où les équilibres physiologiques et psychologiques de l'individu se dérèglent. Dans un contexte professionnel, cet état d'épuisement intervient lorsque le travail rime avec la souffrance.

La souffrance au travail affecte non seulement l'individu qui la vit mais elle interpelle également l'organisation toute entière. Face à une actualité qui regorge d'exemples de salariés en détresse, l'ampleur du phénomène est à présent reconnue. Ce sujet sensible mobilise même l'attention des pouvoirs publics qui décide d'en faire une urgence nationale. Suite aux suicides médiatisés chez France Télécom-Orange et au Pôle Emploi (nouvelle structure issue de la fusion entre l'ANPE et les Assedics), un plan d'urgence a été lancé incitant les entreprises de plus de 1000 salariés à négocier. L'Etat, les syndicats d'employeurs et de salariés, les $\mathrm{DRH}$, mais aussi les petits entrepreneurs ont à leur tour pris conscience de l'ampleur du phénomène. De nos jours, ces dimensions de la vie professionnelle sont englobées sous l'expression « risques psychosociaux » (RPS) qui regroupent à la fois le stress, l'épuisement, les violences, le harcèlement, les TMS..., et qui font l'objet de nombreuses préconisations dans le cadre de l'obligation de prévention qui s'exercent sur les employeurs.

Dans le domaine de la recherche, plusieurs études ont cherché à identifier les causes et les conséquences de l'épuisement professionnel (Neveu, 1995 ; Edey Gamassou, 2006). Certaines se sont focalisées sur les caractéristiques propres à l'individu (Chanlat, 1986). D'autres ont porté leur attention sur l'organisation dans laquelle évolue le salarié. Plus rares sont les recherches qui mettent en lumière l'importance des relations qu'entretient l'individu avec son environnement. Nous pensons en effet que travailler, c'est produire, mais c'est aussi vivre avec les autres. De ce fait, notre problématique cherche à montrer l'existence de liens entre l'environnement relationnel de l'individu et sa propension à être exposé à un épuisement professionnel.

Afin d'apporter des éléments de réponses à notre problématique, notre recherche s'organise en trois grandes parties. La première partie décrit l'importance de l'environnement relationnel d'un salarié. Cet environnement concerne aussi bien ses relations avec l'organisation, ses relations avec l'entourage qu'avec les liens travail/famille. La deuxième partie définit précisément le concept d'épuisement professionnel et les mesures associées. Ces deux concepts d'environnement relationnel et d'épuisement professionnel vont être repris dans la troisième partie pour la description de notre modèle de recherche. La méthodologie est détaillée dans la quatrième partie, avant d'analyser les résultats dans la dernière partie. Enfin, nous concluons par un état d'avancement de notre recherche. 


\section{L'environnement relationnel}

Les relations difficiles avec le public dans certains métiers (infirmières, chauffeur de bus par exemple) peuvent constituer un facteur de stress. Les comportements de clients ou d'usagers sont souvent imprévisibles. De plus, le travail relationnel comme le souligne Loriol (2004), ne produit souvent rien d'autre qu'un service qui disparaît aussitôt rendu et ne se concrétise dans aucun objet matériel. La reconnaissance du travail effectué n'est pas évidente et son déni constitue une source de souffrance.

En revanche, le soutien social et le soutien organisationnel peuvent avoir des effets modérateurs sur des sources de stress ou sur le stress lui-même. En effet selon la théorie de préservation de ressources développée par Hobfoll et Shirom (1993) la possibilité de se tourner vers un tiers permettrait de mieux faire face aux situations de travail difficiles. Pour certains auteurs, la recherche active d'un soutien constituerait même une stratégie d'ajustement c'est-à-dire un moyen de rétablir l'équilibre face à un environnement jugé stressant (Greenglass, 1993). Ces moyens mis en œuvre par l'individu dans le processus du stress sont appelés stratégies d'ajustement dans la revue de littérature française ou coping en anglais. Les effets négatifs des situations de travail jugées très contraignantes sont renforcés par le manque de soutien social. C'est pourquoi, le modèle de Karasek qui comptait au départ deux dimensions, à savoir la latitude décisionnelle -ou autonomie- et la charge de travail, s'est vu ajouté une dimension supplémentaire en 1990 (Karasek et Theorell) : le soutien social (social support). La perception que le travailleur a du soutien social existant au travail aurait un impact positif sur les facteurs de stress (demandes excessives) et le niveau de stress. D'ailleurs une étude (Moisson et Roques, 2005) soulignait l'importance du soutien social au sein d'un service d'urgences et pointait le manque de soutien organisationnel.

\subsection{Les relations avec l'organisation}

Le soutien de l'organisation se définit comme la perception que le salarié a de la manière dont l'organisation valorise ses contributions et se soucie de son bien-être. Rhoades et Eisenberger (2002) définissent le concept comme l'étendue selon laquelle les salariés pensent pouvoir obtenir de l'aide de la part de l'organisation lorsqu'ils rencontrent des situations difficiles.

La mesure de la perception par le salarié du soutien organisationnel est aujourd'hui bien maîtrisée. L'échelle la plus couramment utilisée a été élaborée par Eisenberger et al., (1986). A l'origine, la version comportait 36 items. Eisenberger et ses collègues rapportent un indice de consistance interne (alpha de Cronbach) de .97. Une version plus courte comprenant 17 items a également été proposée. Cette dernière a été traduite en langue française et a été épurée. Quelques items 
évaluent explicitement le souci de bien-être recherché par l'entreprise pour ses salariés sans en préciser la direction (Dumas, Alis et Campoy, 2004). Tandis que, quatre items font référence au sentiment d'être reconnu pour sa contribution à l'efficacité de l'organisation.

\subsection{Les relations avec l'entourage}

A la fin des années 80 , le concept de soutien social a été ajouté à la suite de centaines d'enquêtes réalisées dans les entreprises, principalement sous l'impulsion de Hall et Johnson (1988). Toutes ces enquêtes montrent que le soutien social est le principal facteur d'augmentation du ressort et du dynamisme des travailleurs. Cela n'a rien d'étonnant puisque l'une des caractéristiques de I'homme a toujours été sa capacité, voire sa propension à vivre en groupe. La façon dont les travailleurs se soutiennent mutuellement et la manière dont on arrive à comprendre la hiérarchie dans l'entreprise, détermineront dans une large mesure la transformation effective de la charge de travail en stress au travail. Pour Leavy (1983), le concept de soutien social fait référence à la disponibilité de relations " aidantes » (« helping relations ») et à la qualité de celles-ci. Dès lors, on peut aborder le soutien social sous plusieurs aspects : la présence d'un réseau social important (Amiel-Lebigre et Gognalons-Nicolet, 1993), c'est-à-dire être impliqué, intégré, sentir que l'on peut compter sur ces personnes en cas de besoin et même comptabiliser les personnes constituant ce réseau. D'autres travaux étudient le type de soutien. II existe plusieurs typologies, à savoir donner des conseils ou de l'information, partager des tâches (soutien social instrumental) ou se pencher sur les démonstrations d'empathie (soutien social émotionnel). Certains auteurs ajoutent une troisième dimension : le soutien tangible (Lazarus et Folkman, 1984). II s'agit ici d'évaluer une aide concrète telle que le prêt d'argent, par exemple. Enfin, le soutien social peut aussi être considéré en fonction de la satisfaction du soutien apporté.

De nombreuses études indiquent combien le soutien social est important pour la santé. Dans leur méta analyse, House, Landis, et Umberson (1988) ont montré que le soutien social était associé à une meilleure santé. II apparaît que les individus les plus isolés ou les moins intégrés socialement sont physiquement et psychologiquement en moins bonne santé et qu'ils risquent de mourir plus jeunes. II semblerait donc qu'il y ait une relation forte entre le soutien social, le stress et le bien-être. Cobb propose que le soutien social soit considéré comme de l'information, c'est-à-dire de l'information conduisant la personne à croire qu'elle est appréciée et aimée, qu'elle est estimée et qu'elle fait partie d'un réseau. Ce soutien peut donc être au plan émotionnel, au plan de l'estime de soi ou au plan du sentiment d'appartenance. II est habituellement reconnu que les gens ont besoin de relations stables afin de les protéger contre les risques, en matière de santé, de l'isolement social. 
Après nos diverses entrevues auprès des infirmières, et étant donné la multiplicité potentielle d'acteurs pouvant être sollicités au sein établissements de soins (ASH, aides-soignantes, infirmières d'autres services...), mais aussi dans le cadre du cercle privé (famille, conjoint et amis) nous avons choisi deux échelles du soutien. La distinction s'est faite entre le soutien provenant de l'organisation et de la hiérarchie et celle provenant de l'entourage. En effet, au cours des entretiens, il est apparu important de prendre également en compte ces deux dimensions. Nous avons donc opté pour deux échelles de mesure, nous les présentons ci-après. En outre, il est important de souligner que dans un même environnement de travail les salariés peuvent être en présence de personnes dites « ressources » qui vont apporter du soutien, tandis que d'autres personnes seront sources de conflits. Enfin, nous ne cherchons pas à mesurer, comme Barrera (1986) « le nombre de relations sociales qu'un individu a établies avec autrui, la fréquence des contacts sociaux effectifs avec ces personnes et l'intensité de ces liens ». Cette dimension structurale du soutien décrit le degré relatif d'intégration sociale ou d'isolement d'un individu (Bruchon-Schweitzer, 2002). Nous axons notre recherche sur la source du soutien perçu.

\subsection{Les relations avec le couple travail/famille}

La famille et le travail constituent les deux principales sources qui constituent, à des degrés divers, l'identité des individus (Laufer, 1982). Mais il s'agit aussi de deux valeurs qui sont fortement consommatrices de temps et entre lesquelles il faut parfois arbitrer. Landrieux-Kartochian (2003) note que les relations entre ces deux valeurs sont souvent évoquées au travers du conflit travail-famille. Le conflit vie privée - vie professionnelle peut se définir comme une sorte de conflit inter-rôles dans lequel les pressions de rôles venant du travail et de la famille sont dans une certaine mesure mutuellement incompatibles (Kahn et al., 1964 ; Greenhaus et Beutell, 1985). Un conflit de ce genre a pour résultat deux types de tensions (Duxbury et al., 1999) : la surcharge de rôle (lorsque la quantité de travail est trop importante en regard du peu de temps imparti) et l'interférence des rôles (lorsque des exigences incompatibles rendent difficiles l'accomplissement d'un rôle, par exemple lorsqu'un employé doit être à deux endroits différents au même moment).

Les chercheurs se sont davantage intéressés aux déterminants du conflit vie privée - vie professionnelle qu'à ses effets (St-Onge et al., 2002). Pourtant, les effets négatifs de la perception de conflit travail-famille sur les attitudes et comportements au travail sont nombreux : stress professionnel (Bacharach et al., 1991 ; Guérin et al., 1997), diminution de l'engagement (Frone et al., 1992), augmentation du roulement (McCracken, 2000), augmentation de l'absentéisme et des retards au travail (Paris, 1989)... Les statistiques récentes tendent à montrer que l'articulation vie privée - vie professionnelle est devenue une attente 
majeure des salariés français. Ainsi, selon une étude de la DARES, "parmi les personnes qui exercent une activité professionnelle, près de quatre sur dix trouvent que leur travail rend difficile l'organisation de leur vie de famille » (Garner et al., 2004, p.1). Le secteur des infirmier(e)s connaît des problèmes spécifiques, accentuant ce phénomène général : des horaires de travail contraignants, des changements de plannings, l'alternance du travail jour/nuit, une activité le weekend et les jours fériés rendent difficile l'équilibre entre vie professionnelle et vie privée. On compte en France plus de 3,5 millions d'infirmières dont $73 \%$ pour le secteur public. II s'agit d'un milieu majoritairement féminin $(84,3 \%)$ ce qui exacerbe l'acuité du problème de " conciliation vie familiale / vie professionnelle " puisque les femmes assurent toujours une part bien plus importante que les hommes du travail domestique.

\section{L'épuisement professionnel (Burnout)}

L'épuisement professionnel (ou burnout en anglais) a été décrit initialement dans les professions soignantes dans lesquelles les professionnels sont confrontés quotidiennement à la maladie, à la souffrance et à la mort. Ce sont des professions qui les placent sous l'influence d'une véritable éthique professionnelle (Canoui, 1996). Ensuite, ces professions impliquent des considérations profondément humanistes, souvent idéalisées et parfois loin du travail quotidien (Jaoul et Kovess, 2003). Choisir de travailler dans ces professions est souvent un acte déterminé par une véritable vocation, des idéaux marqués. Or, les soignants pointent une charge de travail de plus en plus lourde entraînant un manque de temps auprès des patients ayant pour conséquence un sentiment de frustration. Par ailleurs, les infirmières ont de plus en plus de responsabilités et doivent remplir de nombreux formulaires pour se " couvrir " du risque d'erreur. Ainsi la charge de travail et la désillusion y sont d'autant plus vécues avec émotion. Dans la langue courante anglaise, burnout signifie "s'user, s'épuiser, craquer en raison de demandes excessives d'énergie, de forces ou de ressources ».

Le concept d'épuisement professionnel a été introduit pour la première fois par le psychanalyste américain Freudenberger (1974). Psychothérapeute, psychiatre, il dirigeait un hôpital de jour alternatif accueillant des toxicomanes au Lower East Side de New York qui fonctionnait principalement à l'aide de jeunes bénévoles. Freudenberger observa un phénomène paradoxal. Beaucoup de ces jeunes finissaient, après un an d'activité, par perdre l'enthousiasme qu'ils avaient au départ. Des symptômes physiques tels que la fatigue, la persistance de rhumes, de maux de tête, de troubles gastro-intestinaux, d'insomnies, d'épuisement caractérisaient ces changements. Colère, irritation, cynisme, attitudes négatives, incapacité à faire face aux tensions mais aussi perte d'énergie sont parmi les premiers signes de ce qu'il nomme " craquage " ou " épuisement émotionnel et mental ». Freudenberger relève aussi des stratégies de surenchère chez son 
équipe, comme par exemple le fait de passer de plus en plus de temps au travail en tournant en rond de façon inefficace, ou, à l'inverse, des stratégies d'évitement en cherchant l'isolement ou en refusant le contact avec les collègues.

D'après Christina Maslach, chercheur en psychologie sociale, le burnout semble commun aux professionnels de santé et aux avocats. De ce constat, elle émit l'hypothèse que travailler avec d'autres, en particulier dans une relation d'aide, est le cœur du phénomène. A l'inverse de Freudenberger qui insistait sur des facteurs personnels, elle situe davantage les causes du burnout dans l'environnement de travail. Elle cherchera à valider cette idée en menant des enquêtes auprès d'autres groupes professionnels dont l'activité suppose aussi une implication relationnelle. Maslach va en 1976 relater les résultats de ses premières investigations en employant très fréquemment dans son article le terme de " craquage ». Elle observe que ce « craquage » est suivi d'une perte d'efficacité dans les services de santé et d'action sociale, d'un absentéisme et d'un turnover élevé. II provoque une détérioration du bien-être physique : "Les professionnels sont épuisés, fréquemment malades et peuvent souffrir d'insomnies, d'ulcères et de maux de tête [...]. Afin de surmonter ces problèmes physiques, le travailleur peut se tourner vers les tranquillisants, la drogue [...].

Le burnout est encore associé à des manifestations comme l'alcoolisme, la maladie mentale, les conflits conjugaux ou le suicide. »

Les travaux de recherche en psychologie sociale réalisés par Maslach et ses collègues (Maslach et Jackson, 1986) ont contribué au développement du concept et à sa reconnaissance au plan scientifique. Selon ces auteurs, l'épuisement professionnel est un syndrome psychologique d'épuisement émotionnel, de dépersonnalisation et de faible accomplissement personnel qui survient principalement dans les professions engagées dans une relation d'aide (Maslach et Jackson, 1986).

- La première composante de l'épuisement professionnel, également nommé l'assèchement émotionnel, fait référence au sentiment d'épuisement et de vide intérieur provoqué par le travail, à l'appauvrissement des ressources émotionnelles et au sentiment de ne plus pouvoir donner à autrui au plan psychologique. Cet état peut coexister avec des sentiments de frustration et de tension ou bien peut se manifester par une absence d'émotion (Hollet, 2006)

- La seconde dimension a trait à la dépersonnalisation vis à vis des clients ou patients. Elle se manifeste par le développement de sentiments et d'attitudes négatifs et cyniques envers la clientèle et peut conduire à la déshumanisation progressive des clients et des interventions. En effet, le travailleur peut tenter d'éviter tout contact et va traiter les patients comme des numéros.

- Enfin, la troisième composante consiste en un faible accomplissement personnel ou perte d'efficacité professionnelle. Lorsque les efforts répétés 
ne produisent plus de résultats, ce sentiment est caractérisé par une impression d'inefficacité, la réduction de la motivation, et une baisse d'estime de soi. Ce sentiment de non-accomplissement personnel est accompagné d'insatisfaction, d'un sentiment d'échec, de fatalité, d'incompétence, d'incapacité à répondre aux attentes de son entourage (Neveu, 2005).

Selon cette conception, l'individu serait affecté en trois temps. II serait tout d'abord fatigué émotionnellement, ainsi pressé d'en finir il développerait un comportement distant et négatif, découlant ensuite sur un sentiment de diminution de son efficacité professionnelle.

En dépit de quelques différences quant à leur conception de l'épuisement professionnel comme un état, un syndrome ou un processus et quant aux comportements qu'elles retiennent comme causes ou effets, ces définitions présentent néanmoins plusieurs constantes et éléments communs (Vanier, 1999).

\section{Le modèle de recherche}

Le modèle de recherche vise à montrer qu'il existe des relations négatives entre l'environnement relationnel de l'individu, dans sa dimension organisationnelle, sociale ou privée et son épuisement professionnel. Notre principale hypothèse de recherche est que l'individu qui se situe à la marge de son environnement relationnel (relation avec l'organisation, avec l'entourage, travail-famille) sera plus exposé au burnout.

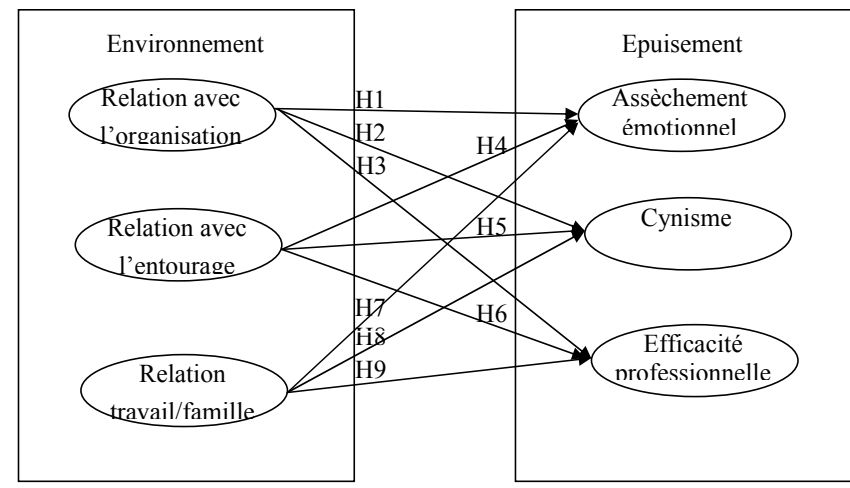

Figure 1 : Le modèle de recherche

Dans le cadre de cette recherche, nous proposons d'évaluer le soutien social perçu des collègues d'une part, de l'organisation et du supérieur d'autre part. En effet, nous avons souhaité mobiliser deux instruments de mesure différents selon la provenance de l'aide apportée dans un contexte professionnel. Selon 
l'hypothèse formulée, un soignant percevant un soutien de la part de ses collègues et de son organisation sera moins exposé aux risques d'épuisement professionnel. Réciproquement, une absence de soutien perçu sera sans doute négativement corrélée avec l'assèchement émotionnel.

Nous formulons les hypothèses suivantes:

$\mathrm{H} 1$ : Il existe une relation négative significative entre les relations organisationnelles de l'individu et son assèchement émotionnel. Plus il a le sentiment d'avoir le soutien de son organisation et moins il sera exposé à l'assèchement émotionnel.

$\mathrm{H} 2$ : Il existe une relation négative significative entre les relations organisationnelles de l'individu et son cynisme. Plus il a le sentiment d'avoir le soutien de son organisation et moins il sera exposé à un sentiment de cynisme.

$\mathrm{H} 3$ : Il existe une relation positive significative entre les relations organisationnelles de l'individu et son efficacité professionnelle. Plus il a le sentiment d'avoir le soutien de son organisation et plus il se sentira efficace professionnellement.

$\mathrm{H} 4$ : Il existe une relation négative significative entre les relations avec l'entourage de l'individu et son assèchement émotionnel. Plus il aura le sentiment d'être soutenu par son entourage et moins il sera exposé à l'assèchement émotionnel.

H5 : Il existe une relation négative significative entre les relations avec l'entourage de l'individu et son cynisme. Plus il aura le sentiment d'être soutenu par son entourage et moins il sera exposé à un sentiment de cynisme.

$\mathrm{H} 6$ : Il existe une relation positive significative entre les relations avec l'entourage de l'individu et son efficacité professionnelle. Plus il a le sentiment d'avoir le soutien de son entourage et plus il se sentira efficace professionnellement.

$\mathrm{H} 7$ : Il existe une relation positive significative entre les relations avec le couple travail-famille de l'individu et son assèchement émotionnel. Plus il aura le sentiment que son travail empiète sur ses responsabilités et rôles familiaux et plus il sera exposé à l'assèchement émotionnel.

$\mathrm{H} 8$ : Il existe une relation positive significative entre les relations avec le couple travail-famille de l'individu et son cynisme. Plus il aura le sentiment que son travail empiète sur ses responsabilités et rôles familiaux et plus il sera exposé à un sentiment de cynisme.

H9 : Il existe une relation négative significative entre les relations avec le couple travail-famille de l'individu et son efficacité professionnelle. Plus il aura le sentiment que son travail empiète sur ses responsabilités et rôles familiaux et moins il se sentira efficace professionnellement.

Ce modèle sera testé selon une méthodologie décrite ci-dessous. 


\section{Méthodologie}

\subsection{La collecte des données}

Avant de diffuser largement le questionnaire, nous avons réalisé un pré-test de notre questionnaire auprès de quelques infirmières. Ces dernières n'ayant pointé aucune difficulté tant au niveau de la compréhension des items que dans leur formulation, nous avons choisi de le diffuser en l'état. Le questionnaire a donc été " auto-administré », c'est-à-dire lu et rempli par l'enquêté lui-même, afin de tenir compte des contraintes de faisabilité technique et financière.

639 questionnaires exploitables ont été retenus pour l'analyse. Le taux de retour s'élève à $25 \%$ ce qui est assez satisfaisant. La moyenne d'âge de notre échantillon est de 38,6 ans, pour une ancienneté moyenne de 7 ans dans le poste, de 11 ans dans l'hôpital et de 14 ans dans le métier. La grande majorité des répondants sont des femmes $(91,2 \%)$. La plupart vivent en couple $(78,5 \%)$ et ont en moyenne 1,3 enfants à charge. Ces chiffres se rapprochent de ceux de la population infirmière française, au regard d'une étude de la DREES (2004). Enfin, les personnes interrogées travaillent dans une grande variété de services hospitaliers : urgences, cancérologie, oncologie, bloc opératoire, médecine, réanimation, pédiatrie, gériatrie, psychiatrie, chirurgie, pneumologie, maternité...

\subsection{Les variables dépendantes}

Plusieurs variables sont disponibles pour décrire le phénomène d'épuisement professionnel. Tout d'abord l'assèchement émotionnel, caractérisé par plusieurs facteurs. Le premier est la sensation d'être " vidé " par son travail. De même, la sensation d'être " usé » à la fin de la journée, à force de travailler caractérise également cette idée d'assèchement émotionnel. Enfin, la pénibilité du travail et la sensation de fatigue continuelle participent également à la caractérisation de cet assèchement émotionnel.

Une autre mesure de l'épuisement professionnel est le cynisme. Ce dernier est caractérisé par le désintérêt par le métier pratiqué, ainsi que par le manque d'enthousiasme dans la pratique du métier. De la même façon, un certain détachement vis-à-vis des autres caractérise également cette idée de cynisme. L'individu se sent perplexe quant à sa contribution dans son entreprise, il doute de l'importance de son travail.

Une dernière mesure de l'épuisement professionnel est l'efficacité professionnelle de l'individu. II s'agit ici d'une efficacité perçue par l'individu lui-même. Elle se traduit par la résolution efficace des problèmes, ainsi que par une contribution efficace de l'individu à son entreprise. L'individu a le sentiment d'agir de façon efficace. II pense être efficace dans son métier, et pense avoir accompli beaucoup 
de choses qui en valent la peine. Cette efficacité professionnelle participe à sa sensation euphorique lorsqu'il accomplit son travail.

\begin{tabular}{|l|l|c|}
\hline Variables dépendantes & \multicolumn{1}{|c|}{ Caractéristiques } & Noms \\
\hline Assèchement émotionnel & Se sentir vidé affectivement & AE1 \\
\cline { 2 - 3 } & Se sentir usé à la fin de la journée & AE2 \\
\cline { 2 - 3 } & Pénibilité du travail & AE3 \\
\cline { 2 - 3 } & Se sentir usé à force de travailler & AE4 \\
\cline { 2 - 3 } & Se sentir fatigué pour affronter une nouvelle journée & AE5 \\
\cline { 2 - 3 } & Manque d'intérêt pour le métier & C1 \\
\cline { 2 - 3 } & Manque d'enthousiasme pour son métier & C2 \\
\cline { 2 - 3 } & Détachement par rapport aux autres & C3 \\
\cline { 2 - 3 } & Perplexité quant à sa contribution pour son entreprise & C4 \\
\cline { 2 - 3 } & Doute de l'importance de son travail & C5 \\
\cline { 2 - 3 } & Résolution efficace des problèmes & EP1 \\
\cline { 2 - 3 } & Contribution efficace à l'organisation & EP2 \\
\cline { 2 - 3 } & Bonne pratique du métier & EP3 \\
\cline { 2 - 3 } & Sensation d'euphorie dans la pratique du métier & EP4 \\
\cline { 2 - 3 } & Accomplissement d'activités valables & EP5 \\
\cline { 2 - 3 } & Agissement efficace au travail & EP6 \\
\hline
\end{tabular}

Tableau 1 : Récapitulatif des variables dépendantes

\subsection{Les variables indépendantes}

Plusieurs variables sont monopolisées pour décrire l'environnement relationnel de l'individu. Tout d'abord, ses relations par rapport à son organisation. II s'agit ici d'évaluer dans quelle mesure il a le soutien de son organisation. Ce soutien peut se caractériser par le fait que l'organisation apprécie la contribution que l'individu apporte à la réussite de l'entreprise. II se caractérise également par la prise en compte des aspirations et des valeurs de l'individu. Ce soutien peut prendre la forme d'une aide de la part de l'entreprise lorsque l'individu a un problème, d'une aide pour que l'individu puisse faire son travail au mieux de ses capacités. L'organisation est même prête à aider l'individu lorsque celui-ci a besoin d'une faveur spéciale. Elle soutien l'individu en se préoccupant de sa satisfaction générale au travail et essaie de rendre son travail le plus intéressant possible. Enfin, elle prend en compte les opinions de l'individu et apprécie ses réalisations au travail.

L'environnement relationnel de l'individu est également caractérisé par ses relations avec son entourage. II s'agit ici d'évaluer son soutien social. Le soutien social peut se manifester par un réconfort, une écoute, un conseil lorsque l'individu a des soucis ou lorsque les problèmes surviennent au travail. L'entourage s'occupe de l'individu et l'aide également matériellement. Enfin, l'entourage est présent pour que l'individu puisse se confier et parler librement de ses problèmes. 
Les relations travail-famille caractérisent également l'environnement relationnel de l'individu. En général, ces relations travail-famille sont conflictuelles. Autrement dit, il s'agit ici d'évaluer dans quelle mesure l'individu arrive à concilier ces deux contraintes (familiales et professionnelles). Le conflit peut provenir des horaires de travail incompatibles. Le travail nécessite autant de temps que l'individu ne peut pas passer en famille, son travail empiète sur le temps qu'il aimerait consacrer à sa famille. Une sensation de fatigue trop intense au travail peut empêcher d'accomplir certaines tâches à la maison. L'excès de travail en entreprise se fait au détriment des activités personnelles. L'importance donnée au travail fait que la famille se plaint, que l'individu lui-même se trouve irritable et n'arrive pas à se détendre.

\begin{tabular}{|c|c|c|}
\hline $\begin{array}{c}\text { Variables } \\
\text { indépendantes }\end{array}$ & Caractéristiques & Noms \\
\hline \multirow{10}{*}{$\begin{array}{l}\text { Relations } \\
\text { Organisationnelles }\end{array}$} & Contribution appréciée par l'organisation & SOP1 \\
\hline & Aspirations et valeurs prises en compte par l'organisation & SOP2 \\
\hline & Aide de la part de l'organisation en cas de problème & SOP3 \\
\hline & L'organisation se préoccupe du bien-être & SOP4 \\
\hline & Aide de l'organisation pour bien faire le travail & SOP5 \\
\hline & Aide de l'organisation pour obtenir une faveur & SOP6 \\
\hline & L'organisation se préoccupe de la satisfaction & SOP7 \\
\hline & L'organisation prend en compte les opinions & SOP8 \\
\hline & L'organisation est fière des réalisations & SOP9 \\
\hline & L'organisation tente de rendre le travail intéressant & SOP10 \\
\hline \multirow[t]{6}{*}{ Relations Entourage } & Réconfort et écoute de l'entourage & SS1 \\
\hline & Aide matériel de l'entourage & SS2 \\
\hline & Conseils de l'entourage & SS3 \\
\hline & Réconfort de l'entourage en cas de soucis & SS4 \\
\hline & Possibilité de se confier à l'entourage en cas de problème & SS5 \\
\hline & Aide de l'entourage si difficulté au travail & SS6 \\
\hline \multirow[t]{8}{*}{ Relations Travail-Famille } & Conflits horaires & WIF1 \\
\hline & Trop de fatigue au travail pour d'autres activités choisies & WIF2 \\
\hline & Trop de travail au détriment d'activités personnelles & WIF3 \\
\hline & Plainte de la famille car trop préoccupé par le travail & WIF4 \\
\hline & Irritable car trop accaparé par le travail & WIF5 \\
\hline & Non détendu car travail trop exigeant & WIF6 \\
\hline & Travail empiète sur le temps familial & WIF7 \\
\hline & Travail rend difficile le rôle familial souhaité & WIF8 \\
\hline
\end{tabular}

Tableau 2 : Récapitulatif des variables indépendantes 


\subsection{Le traitement des données}

\subsubsection{Le modèle de mesure}

Le traitement des données se fait en plusieurs étapes. La première a une visée confirmatoire. Dans ce but, une analyse factorielle des différentes mesures présentées est effectuée. Pour cela, nous avons utilisé le logiciel PASW Statistics 18 de SPSS. Cette analyse permet de détecter la présence d'un ou plusieurs facteurs communs significatifs. Afin de tester l'adéquation de l'échantillon, le critère Kaiser, Meyer, Olkin (KMO), est utilisé (celui-ci doit être supérieur à 0,5). Le test de sphéricité de Bartlett est utilisé pour savoir si la matrice de covariance est significativement différente de la matrice unité. Un test du Khi-deux est effectué et le déterminant analysé. Pour identifier les facteurs, la méthode du maximum de vraisemblance est utilisée. Cette méthode est robuste, même si les données ne sont pas distribuées normalement. Pour vérifier le nombre de facteurs, le critère de Kaiser est utilisé (valeurs propres supérieures à 1). Le cas échéant, une rotation est effectuée avec la méthode varimax pour identifier les mesures initiales. La fiabilité de la mesure est donnée par le calcul de l'alpha de Cronbach. On s'assure ainsi de la validité convergente et de la validité discriminante.

\subsubsection{Le modèle structurel}

Le modèle structurel a été testé avec le logiciel LISREL (version 8.80) en utilisant la méthode du maximum de vraisemblance. Pour mesurer l'adéquation du modèle aux données, plusieurs critères peuvent être utilisés (voir [Jöreskog and Sörbom, 1993, 2001], pour des informations détaillées).

Le modèle structurel initial a été construit de façon à identifier et évaluer les relations entre l'environnement relationnel d'une part et le burnout d'autre part. Cependant, selon les indicateurs d'ajustement décrits ci-avant, le modèle initial ne convergeait pas suffisamment. Des corrections ont été apportées en fonction des résultats de LISREL (indices de modification, changement de la valeur du Khi deux, observation de la matrice de covariance/corrélation, observation des résidus...) dans le but d'améliorer les indicateurs d'ajustement. Le résultat final est décrit ci-après.

\section{Les résultats}

\subsection{Adaptation du modèle}

Le modèle de recherche s'attache à identifier les effets de l'environnement relationnel sur le comportement des personnes. L'environnement relationnel se caractérise par les relations avec l'entourage (ou soutien social), les relations avec l'organisation (ou soutien organisationnel) et les relations «Travail-Famille » 
traduisant les interactions entre les sphères intime et professionnelle. En ce qui concerne le comportement individuel, il est interprété du point de vue de l'épuisement professionnel (« burnout »). Dans ce but, des échelles obtenues à partir de la littérature ont été utilisées : l'assèchement émotionnel, le cynisme et l'efficacité professionnelle du point de vue du répondant.

Lors de l'utilisation de ces échelles, une procédure de contrôle de validité a été mise en œuvre. Globalement, à quelques aménagements près, les échelles «Assèchement émotionnel » et « Efficacité professionnelle » ont pu être utilisées directement. Ce n'est pas le cas pour l'échelle associée au « Cynisme ». Lors de la procédure de factorisation, l'observation des résultats (test de Kaïser, loadings, résidus, R2...) montre que les indicateurs explicites ne convergent pas vers un seul facteur mais plutôt vers deux facteurs. Le premier, que nous appellerons " Cynisme-Enthousiasme » (CynEnt) correspond aux questions C1 (" Je suis moins intéressé(e) par mon métier depuis que je suis dans cette entreprise ») et C2 (" Être infirmier(e) me rend moins enthousiaste »). En observant l'intitulé des questions, nous remarquons une orientation sur le métier (d'infirmier). Le second "Cynisme-Doute » (CynDoute) correspond aux questions C4 (" Je suis devenu(e) plus perplexe quant à ma contribution pour cette organisation ») et C5 (« Je doute parfois de l'importance de mon travail »). Le facteur associé ayant pour " loading » principal l'indicateur C4, nous constatons une orientation sur le doute relatif à l'organisation. Nous observerons dans les résultats que ces facteurs n'interviennent pas de la même façon dans le modèle testé.

Par rapport au modèle initial et compte tenu des résultats de LISREL, des corrections ont été apportées. Ainsi, les indicateurs WIF1, WIF5, WIF8, C3, EP1, EP2, EP4, SOP1 ont été supprimés car leur $\mathrm{R}^{2}$ est trop faible ; ces indicateurs partagent peu de variance avec leur facteur. Nous avons également autorisé une erreur de covariance entre SS6 et SS5, indicateurs d'une même échelle de mesure, afin d'améliorer l'ajustement. Dans le modèle final, tous les loadings sont significatifs (loadings $>0.7$ ).

De plus, en analysant la matrice de corrélation des variables dépendantes, les résultats affichent une très forte corrélation entre les variables du cynisme et l'assèchement émotionnel. De la même façon, les résultats affichent une forte corrélation entre la relation travail-famille (RELWF) et l'assèchement émotionnel (ASSEMO). Nous avons alors ajouté une relation entre ces concepts. Enfin, les résultats affichent une forte corrélation négative entre l'assèchement émotionnel (ASSEMO) et la perception de l'efficacité professionnelle (EFFPRO). Nous ajoutons également une relation entre ces concepts. Les paragraphes ci-après décrivent le modèle structurel final. 


\subsection{Le modèle structurel final}

Le modèle final obtenu converge de façon acceptable même si certains critères ne sont pas dans les valeurs "standards ". Ainsi, le Chi-Deux est élevé mais la sensibilité de cet indicateur à la taille de l'échantillon est connu (il conduit à rejeter les modèles testés sur des échantillons de plus de 200 observations). De plus, les indicateurs GFI et AGFI ont des valeurs faibles mais il est connu que leur valeur est sensible à la complexité des modèles et pénalise ceux ayant beaucoup de paramètres à estimer (Roussel et al., 2002).

\begin{tabular}{|l|c|c|}
\hline & Résultats obtenus & Résultats acceptables \\
\hline Degrees of Freedom & 389 & \\
\hline Chi-Square & 1609 & \\
\hline X $^{2} / \mathrm{df}$ & 4,13 & $<5$ \\
\hline P & 0,0 & $<0,08$ \\
\hline RMSEA & 0.076 & $<0,05$ \\
\hline RMR & 0.048 & $>0,9$ \\
\hline NFI & 0.95 & $>0,9$ \\
\hline NNFI & 0.95 & $>0,9$ \\
\hline CFI & 0,96 & $>0,9$ \\
\hline GFI & 0.84 & $>0,9$ \\
\hline AGFI & 0.80 & \\
\hline
\end{tabular}

Tableau 3 : Critères d'adéquation du modèle

Considérant la complexité du modèle et la taille de l'échantillon, nous pouvons considérer que le modèle décrit dans le schéma ci-après traduit correctement les relations entre les indicateurs de l'échantillon.

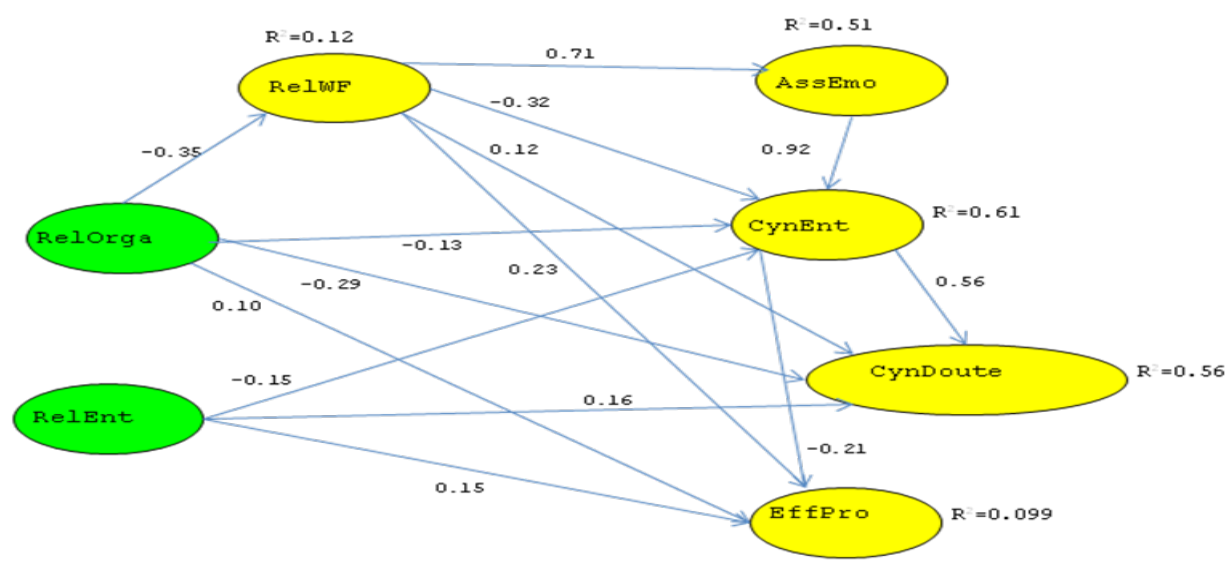

Chi-Scruare $=1609, d f=389, P$-value $=0.00000$, RMSEA $=0.076$

Figure 2 : Le modèle structurel final 
Globalement, les résultats montrent des relations significatives : l'environnement relationnel a un effet sur l'épuisement professionnel des individus. Toutefois, il est nécessaire de nuancer ce propos général et de préciser la nature de ces relations.

En ce qui concerne l'environnement relationnel, deux variables sont indépendantes dans le modèle : les Relations Organisationnelles ou Soutien organisationnel (RelOrga) et les Relations avec l'Entourage ou Soutien Social (RelEnt). La troisième variable, la Relation Travail-Famille (RelWF) traduit le conflit potentiel entre la vie professionnelle et la vie personnelle. La première équation structurelle montre que la relation Travail-Famille est significativement influencée par le soutien organisationnel (coeff. $=-0,35 ; t=-7,74$ ). Plus l'individu a le sentiment d'être soutenu par son organisation, moins il ressent le conflit entre les univers professionnel et personnel.

En ce qui concerne les effets associés au burnout, trois variables ont été prévues par la démarche exploratoire initiale : la fatigue (AssEmo : Assèchement émotionnel), le cynisme et l'efficacité professionnelle. La fatigue (AssEmo) est significativement influencée par le conflit Travail-Famille (coeff. : 0,71, $t=14,67$ ). Plus le conflit Travail-Famille est perçu, plus la fatigue est ressentie par l'individu. De plus, les résultats montrent que la fatigue est majoritairement expliquée par cette variable conflit Travail-Famille $\left(R^{2}=0,51\right)$. Enfin, il est à noter que les autres variables de l'environnement relationnel, les soutiens organisationnel et social, n'ont pas d'effet sur la fatigue.

Le cynisme associé au manque d'enthousiasme (CynEnt) et le cynisme associé au doute (CynDoute) ne sont pas influencés de la même façon par les variables relationnelles. Avec un pourcentage de variance expliquée de $61 \%\left(R^{2}=0,61\right)$, les variables du modèle expliquent majoritairement le manque d'enthousiasme (CynEnt). La fatigue (AssEmo) influence positivement le manque d'enthousiasme (coef. :0,92; $t=12,60$ ). Le manque d'intérêt et d'enthousiasme au travail sont donc étroitement associés à la fatigue. De plus, toutes les variables de l'environnement relationnel ont des effets significatifs. Plus le soutien organisationnel est perçu (coeff. : - 0,13 ; $\mathrm{t}:-3,27$ ), moins l'individu manque d'enthousiasme. Plus le soutien social (RelEnt) est marqué (coeff. : $-0,15 ; t:-3,94$ ), moins l'individu manque d'enthousiasme. Les soutiens social et organisationnel entretiennent d'une certaine façon l'enthousiasme des individus.

De façon plus originale, le conflit Travail-Famille (RelWF) est négativement associé au manque d'enthousiasme. Autrement dit, plus un individu perçoit le conflit Travail-Famille, plus il est intéressé et enthousiaste. Cela peut s'interpréter en considérant le caractère dual du conflit Travail-Famille. Pour qu'un conflit soit ressenti par un individu, il doit à la fois poursuivre des objectifs personnels d'une part, et des objectifs professionnels d'autre part. En cas de conflit Travail- 
Famille avéré, l'engagement au niveau professionnel n'est donc pas à remettre en cause, au contraire. C'est parce que l'individu souhaite rendre compatible son engagement professionnel et sa vie personnelle que le conflit est ressenti plus ou moins vivement. Si l'engagement au niveau professionnel est très marqué, le conflit Travail-Famille sera d'autant plus perçu. En conséquence, le conflit Travail-Famille en tant qu'indicateur d'un engagement professionnel marqué est négativement et significativement associé à un manque d'intérêt/enthousiasme dans l'exercice du métier d'infirmier (Coeff. : $-0,32 ; \mathrm{t}:-5,03$ ).

Notons que cette variable "Conflit Travail-Famille " a un effet inverse sur le cynisme associé au doute (CynDoute) (Coeff. :0,12; $\mathrm{t}: 2,61$ ). Plus le conflit TravailFamille est important, plus le doute apparaît sur la contribution à l'organisation. Ce conflit se traduit donc plus dans la relation à l'organisation. Cette interprétation est confortée par l'effet du Soutien Organisationnel (RelOrga) qui est deux fois plus important pour cette dimension du cynisme. Alors que le coefficient est de -0,13 pour CynEnt, il est de -0,29 dans le cas du doute (Coeff. :-0,29; t : -6,93). Sans surprise, plus le soutien organisationnel est perçu, moins l'individu doute de son apport à l'organisation. En revanche, le soutien social (RelEnt) a un effet inverse par rapport à la dimension intérêt dans le métier (CynEnt). Avec un coefficient de 0,16 (Coeff. :0,16; $t: 4,15$ ), plus le soutien social est important, plus le doute est important. Cela peut s'interpréter du point de vue du comportement des individus en cas de doute où l'entourage est sollicité pour répondre aux interrogations des acteurs dans l'organisation. Enfin, le manque d'intérêt dans le métier (CynEnt) influence positivement (Coeff. : 0,56; $t: 11,08)$ le doute quant à la contribution dans l'organisation. Notons que les variables relationnelles (Relation Travail-Famille (RelWF), Soutien organisationnel (RelOrga), Soutien social (RelEnt)) et le manque d'enthousiasme dans le métier (CynEnt) expliquent une part significative de la variance $\left(R^{2}=0,56\right)$ de la variable associée au doute dans l'organisation (CynDoute).

Enfin, la dernière variable concerne l'efficacité professionnelle perçue. Même si les variables présentent des effets significatifs, elles expliquent relativement peu ce concept $(\mathrm{R} 2=0,099=9,9 \%)$. Les soutiens social (Coeff. : 0,$15 ; t: 3,14$ ) et organisationnel (Coeff. : 0,10; $t: 1,99$ ) influencent positivement l'efficacité professionnelle perçue. Plus le soutien est important, plus l'acteur a le sentiment d'être efficace dans son travail. Ici aussi, la relation Travail-Famille trouve un écho particulier. En effet, plus le conflit Travail-Famille est ressenti, plus l'individu a le sentiment d'être efficace professionnellement. Cela conforte l'interprétation précédente considérant que l'individu ressentant un conflit Travail-Famille est quelqu'un de professionnellement très investi dans son métier (voir commentaires sur le cynisme enthousiasme CynEnt). Son investissement dans son métier se traduit par un conflit avec la vie de famille mais également par un sentiment accru d'être efficace au travail. Notons que le manque d'enthousiasme et d'intérêt dans le métier s'accompagne d'une baisse de l'efficacité professionnelle perçue 
(Coeff. :-0,21; $\mathrm{t}:-3,59)$. Logiquement, l'autre dimension du cynisme, la relation de doute dans la contribution à l'organisation, n'a pas d'effet significatif sur l'efficacité professionnelle perçue.

\subsection{Conclusion}

\subsubsection{Synthèse}

Les résultats de cette recherche permettent de mieux interpréter les effets de l'environnement relationnel sur le burnout. Les hypothèses reliant l'environnement relationnel aux dimensions du burnout ne sont pas infirmées par les données de l'échantillon. Au-delà de ce résultat général, les dimensions du burnout, la fatigue ou assèchement émotionnel, le cynisme et l'efficacité professionnelle, entretiennent des relations différenciées avec l'environnement relationnel. Les deux premières dimensions ont un niveau d'explication significatif $\left(R^{2}>50 \%\right)$ alors que pour la dernière, l'efficacité professionnelle, le niveau est faible.

En synthèse sur la fatigue (ou assèchement émotionnel), seule la relation TravailFamille exerce une influence significative. Plus le conflit est perçu, plus la fatigue est ressentie. Le soutien social et le soutien organisationnel ne jouent pas de rôle significatif.

En synthèse sur le cynisme, il faut noter les deux dimensions émergentes associées à ce concept : la dimension intérêt dans le métier et celle associée à la contribution à l'organisation (entreprise). Le lecteur peut observer un niveau d'explication relativement élevé $\left(R^{2}=0,60\right.$ et 0,56$)$ avec les variables relationnelles et fatigue (AssEmo). De plus, les variables « conflit Travail-Famille » et « soutien social » exercent des influences asymétriques selon les dimensions considérées. La relation (de conflit) Travail-Famille est négativement associée au manque d'intérêt et d'enthousiasme au travail; En d'autres termes, plus il y a conflit et plus il y a d'intérêt et d'enthousiasme. C'est l'inverse pour le doute dans l'organisation : plus il y a conflit et plus il y a doute. Idem pour le soutien social. Plus le soutien social de l'entourage se manifeste, plus il y a intérêt dans le métier et doute en l'organisation. De plus, le soutien organisationnel perçu exerce des effets significatifs limitant le désintérêt dans le métier et le doute dans l'organisation. Enfin, les résultats montrent des relations étroites entre le manque d'enthousiasme et la fatigue d'une part et le manque d'enthousiasme et le doute dans l'organisation d'autre part.

En synthèse sur l'efficacité professionnelle, si toutes les variables relationnelles exercent un effet positif significatif, le niveau global d'explication est faible. D'autres aspects devraient être mobilisés pour interpréter le phénomène. 
De façon globale, des recherches doivent être menées plus avant pour mieux spécifier certains concepts (cynisme en particulier), distinguer les relations et leur sens entre des concepts qui peuvent être relativement proches.

\subsubsection{Intérêts managériaux}

Les résultats de cette recherche trouvent plusieurs utilisations d'un point de vue managérial. C'est l'objet même de cette recherche de montrer en quoi l'environnement relationnel des individus exerce une influence sur le niveau de burnout. De ce point de vue, le burnout vécu par un individu est influencé par les relations qu'il entretient avec son entourage (soutien social), avec l'organisation (soutien organisationnel) et avec sa famille (relation/conflit Travail-Famille). Le burnout n'est donc pas un processus purement endogène, centré sur l'individu. L'influence caractérisée de son environnement montre qu'il s'agit plutôt d'un phénomène systémique, émergeant des interactions de l'individu avec son environnement. Si les ressorts psychologiques de l'individu peuvent échapper aux actions des managers, ces dernières peuvent s'exercer dans le champ de l'environnement relationnel en général et du soutien organisationnel en particulier. Cela peut prendre la forme d'actions spécifiques associées à des modes de management (management participatif, marques d'attention ...) pour développer le soutien organisationnel. D'autres actions peuvent également être mises en œuvre pour diminuer les tensions entre Travail et Famille en aménageant les conditions de l'exercice professionnel pour mieux intégrer les contraintes individuelles et familiales. C'est notamment le cas de certaines organisations qui développent des pratiques facilitant la gestion du couple travail/famille par la mise en œuvre de crèches en entreprise, pressing (GRH à la carte) (Colle, 2005).

\subsubsection{Intérêts et pistes pour la recherche}

D'un point de vue académique, cette recherche montre le caractère émergent du processus de burnout. Certains concepts sont au cœur de la dynamique. On y remarque en particulier le caractère central de la relation travail-famille, signifiant une volonté d'assumer simultanément son engagement professionnel et personnel. Cette relation s'exprime de façon inverse dans les rapports au métier d'une part et à l'organisation d'autre part (Cynisme). Si le conflit Travail-Famille est associé à l'intérêt dans le métier, il s'exprime dans la relation à l'organisation, augmentant le doute quant à la contribution à l'organisation. Ce rôle du conflit Travail-Famille a pu être interprété en identifiant le caractère hétérogène du cynisme. En effet, la mesure utilisée du cynisme n'est pas vraiment homogène et deux dimensions apparaissent : la dimension intérêt dans le métier et celle de la contribution à l'organisation. Dans son processus de burnout, l'individu semble distinguer ses relations au métier et ses relations à l'organisation ou entreprise lui permettant de l'exercer. Cette distinction obtenue à partir d'un travail exploratoire permet de mieux identifier les dimensions du burnout et les effets de l'environnement relationnel sur ce processus. Ceci est d'autant plus utile que les dimensions de l'environnement relationnel, telles que définies dans 
cette recherche, n'influencent pas de la même façon les différentes dimensions du cynisme. La complexification du modèle de recherche devrait permettre une interprétation plus fine du processus.

Ces résultats ouvrent des perspectives de recherche. La première concerne la caractérisation du cynisme. L'approche exploratoire a permis d'interpréter l'échelle utilisée selon 2 dimensions : la dimension intérêt au métier et la dimension contribution à l'organisation. Des travaux complémentaires devraient permettre de préciser ces dimensions du burnout en développant des échelles de mesure associées. Une autre perspective concerne la recherche de variables influençant la perception de l'efficacité professionnelle. En effet, le niveau d'explication associé au modèle étant faible, il est nécessaire de rechercher de nouvelles variables explicatives. Enfin, une autre perspective concerne une meilleure caractérisation de l'environnement relationnel. Cela nécessite l'usage de nouvelles données, en provenance d'autres terrains, en se basant sur des données enrichies (ex: relations à soi-même (confiance en soi, image de soi, conformité à ses valeurs et principes...), environnement relationnel métier, environnement relationnel organisation, environnement relationnel famille, amis...) et/ou des approches différentes (ex: analyse des réseaux sociaux).

\section{Bibliographie}

Amiel-Lebigre F. et Gognalons-Nicolet M. (1993), Entre santé et maladie, P.U.F. les champs de la santé, Paris.

Bacharach, S.B., Bamberger, P. \& Conley, S. (1991). "Work-Home conflict among nurses and engineers: mediating the impact of role stress on burnout and satisfaction at work ". Journal of Organizational Behavior, 12, 39-53.

Bruchon-Schweitzer M. (2002), Psychologie de la santé : modèles, concepts et méthodes. Liège:Dunod

Canoui P. (1996), Approche de la souffrance des soignants par l'analyse du concept de l'épuisement professionnel, le burn out. Considérations psychologiques et éthiques en réanimation pédiatrique.

Chanlat J.-F. (1986), «Le stress et la santé des cadres féminins : un premier bilan», Gestion. Revue internationale de gestion, 11 (4), 53-61

Colle, Rodolphe (2005) L'amélioration du bien-être des salariés par les temps de travail à la carte, Actes de la première journée de recherche IREGE/AGRH, 7 et 8 avril 2005 Annecy.

Cordes C.L. et Dougherty T.W. (1993), «A Review and an Integration of Research on Job Burnout», Academy of Management Review, 18(4), 621-656

Dumas M. Alis D. et Campoy E. (2004), « L'apport des méthodes d'équations structurelles dans les études longitudinales d'analyse du changement attitudinal. Le cas de l'implication organisationnelle affective et de la perception du soutien organisationnel dans un contexte de changement du temps de travail. Actes AGRH, 2004.

Duxbury, L., Higgins, C. \& Johnson, K.L. (1999). " Un examen des répercussions et des coûts du conflit travail-famille au Canada ". Rapport de l'Agence de Santé Publique du Canada.

Edey Gamassou C. (2006), Stress et implication des agents territoriaux : une approche en termes de ressources. Thèse de doctorat, Université Paris 1 Panthéon Sorbonne. 
Freudenberger H.J. (1974), «Staff burn out», Journal of Social Issues, 30(1), 159-165. Frone, M.R., Russell, M. \& Cooper, L.M. (1992). « Antecedents and Outcomes of WorkFamily Conflict: Testing a Model of the Work-Family Interface ». Journal of Applied Psychology, 77 (1), 65-78.

Garner, H., Méda, D. \& Sénik, C. (2004). « La difficile conciliation entre vie professionnelle et vie familiale ». DARES, Premières Synthèses, $n^{\circ} 50.3$.

Golembiewski R.T., Munzenrider R.F. et Stevenson J.G. (1986), Stress in Organizations Toward a Phase Burn Out Model, Greenwood Press, Praeger Publishers, 284 p.

Greenglass E.R (1993), The contribution of social support to coping strategies, Applied Psychology : an international Review, 42, 323-340.

Hobfoll S. E. et Shirom A. (1993), Stress and burnout in the workplace -conservation of ressources-, dans Golembiewski, Handbook of Organizational Behavior, Marcel Dekker inc., New York, Basel, Hong Kong, 41-60.

Hollet T-H. (2006), " Une application du modèle de déséquilibre " effort-récompense » à la compréhension des comportements de retrait des commerciaux ", $18^{\text {èmes }}$ Journées Nationales des IAE, Montpellier.

House, J.S., R.L. Kahn, J.D. Mc Leod et D. Williams (1985), « Measures and Concepts of Social Support «, dans S. Cohen et S.L. Syme (dir.), Social Support and Health, Academic Press Inc., p. 83-108.

Jaoul et Kovess (2003), Le burnout dans la profession enseignante, annales médico psychologiques, revue psychiatrique, volume 162.

Jöreskog K. G., Sörbom D. (1993), LISREL® 8: Structural Equation Modeling with the SIMPLIS Command Language, Second Edition, Scientific Software International, Inc. Jöreskog K. G., Sörbom D. (2001), LISREL® 8: User's Reference Guide, Second Edition, Scientific Software International, Inc.

Kahn R.L., Wolfe D.M., Quinn R., Snoek J.D., Rosenthal R.A. (1964), Organizational stress, New York: Wiley.

Karasek R.A et Theorell (1990), Healthy work, Stress, Productivity and the Reconstruction of Working Life, New York, The Free Press, Basic Books

Karasek, R.A. (1979), «Job demands, Job decision Latitude and Mental Strain: Implications for Job Redesign », Administrative Quarterly, 24, 285-304

Landrieux-Kartochian, S. (2003). "La GRH au cœur de l'articulation vie privée - vie professionnelle, une étude exploratoire sur les politiques "family friendly » en France ». Actes de la $2^{e}$ Journée thématique AGRH sur la gestion des carrières, Aix-en-Provence, p.30-38.

Laufer, J. (1982), La féminité neutralisée ? Femmes cadres dans l'entreprise. Flammarion, Paris.

Lazarus R.S. et Folkman S. (1984), Stress, appraisal and coping, Springer publishing company, New York.

Leavy, R.L. (1983). Social support and psychological disorder: A review. Journal of Community Psychology, 11, 3-21.

Loriol M. (2004), Quand la relation devient stressante, difficultés et adaptations lors du contact avec les usagers, Humanisme et entreprise, 262, 1-18.

Maslach C. \& Leiter M.P. (2008), Early Predictors of Job Burnout and Engagement, Journal of Applied Psychology, 93(3), 498-512

Maslach C. (1982), Burnout: The cost of caring, New York, Prentice Hall Press.

Maslach C. et Jackson S.E. (1986), The Maslach Burnout Inventory, Manual, Palo Alto, CA : Consulting Psychologists Press.

McCracken, D.M. (2000). "Winning the talent war for women: Sometimes it takes a revolution ». Harvard Business Review, 78 (6), 159-167.

Neveu J.-P. (1995,) «Épuisement professionnel : Concept et Méthodologie», Le Travail Humain , 58(3), 209-228. 
Paris, H. (1989). " Les programmes d'aide aux employés qui ont des obligations familiales ». Conférence Board du Canada, Rapport 43.

Rhoades L. et Eisenberger R. (2002), Perceived organizational support: A review of the literature, Journal of Applied Psychology, vol.84, $n^{\circ} 4$, p.698-714.

Roussel P., Durrieu F., Campoy E., El Akremi A. (2002), Méthodes d'Équations Structurelles : Recherche et Applications en Gestion, Ed. Economica, 276 p.

Selye H. (1975), le stress de la vie-le problème de l'adaptation, nouvelle édition, NRF, Gallimard

St-Onge, S., Renaud, S., Guérin, G. \& Caussignac, E. (2002), « Vérification d'un modèle structurel à l'égard du conflit travail-famille ». Relations Industrielles, 57 (3), 491-516. 